moreover, highly effective in stubborn cases which have proved resistant to the usual methods of treatment.

From the bacteriological viewpoint, penicillin, as employed in this investigation, eradicates sensitive organisms from the conjunctiva in a few days. It cannot, however, prevent reinfection after the cessation of treatment, and we saw no reason to believe that effective therapy with penicillin established any immunity to recurrent infection. In this connection, it is noteworthy that in the cases of this series which showed bacteriological relapse, the organisms causing the relapse were as sensitive to penicillin as were those causing the original infection.

This investigation was sponsored by the Consulting Ophthalmologist to the Army in collaboration with the Director of Pathology.

We wish to thank Colonel O. J. O'B. O'Hanlon and Colonel C. D. M. Buckley, M.C., for providing facilities for the investigation, and Captain A. Stanworth for his assistance with the clinical side of the work.

\title{
REFERENCES
}

1. Abraham, E. P., Chain, E., Fletcher, C. M., Florey, H. W., Gardner, A. D., Heatley, N. G. and Jennings, M. A.-Lancet 2, p. 177, 1941.

2. Fleming, A.-Lancet 2, p. 434, 1943.

3. FlOREY,' M. E. and FloREY, H. W.-Lancet 1, p. 387, 1943.

4. HARPER, G: J.-Lancet 2, p. 569, 1943.

\section{THE INCIDENCE OF EYE DISEASE IN THE AUSTRALIAN IMPERIAL FORCES, MIDDLE EAST*}

BY

\section{Major J. BRuCe Hamilton, A.A.M.C.}

IN the Australian Medical Journal of January 10, 1942, I published for future medical historians a survey of 6,458 private patients. seen in Tasmania between 1931-1939, with 14,317 diseased conditions under 239 disease headings. This gave a ratio of $2 \cdot 22$ diseases per patient. At the conclusion I promised a similar table of eye disease in the Australian Imperial Forces, and now I have pleasure in submitting it. This A.I.F. survey deals with 3,638 patients seen during 1941-1942, with 5,650 diseased conditions under 226 disease headings. This gives a ratio of 1.55 diseases 
per patient: For simplicity I have arranged the figures and percentages of civil and war eye disease side by side in the five tables presented, and for convenience have grouped certain diseases together in four tables. Table No. I is a general survey; in Table II are found the eye injuries; in Table III the visual disorders; in Table IV the muscular abnormalities; and in Table V the keratitis cases. These seemed to be the four groups worthy of special comparative study.

Tables are unsatisfactory reading at all times, so $I$ feel that some generalised comments will be more stimulating. Can it be possible that in war there is less eye disease than in civil life? Definitely it appears so from those figures quoted in paragraph one. Certainly there are less eye injuries, despite the fact that these figures contain casualties from three active campaigns of the A.I.F. Table II shows the civil eye injuries as $15 \cdot 6$ per cent. and the army injuries as $9 \cdot 4$ per cent. On the medical side iritis, keratitis, choroiditis, and retinitis are also lower.

Mentioning iritis makes me emphasise the fact that despite the high incidence of gonorrhoea and dysentery in the Middle East, there is not one case of iritis in these figures from either disease. Interstitial keratitis showed a 0.16 per cent. incidence, while gonococcal ophthalmia has not occurred. It only stresses the fact that disease treatment in the A:A.M.C. is of the very highest order and complications at the very lowest ebb.

The loss of an eye is even lower in military than in civil practice, and so apparently is traumatic iris prolapse and traumatic cataract (see Table II). Vitreous haemorrhage is higher, but this is due to blast effect in battle, and is analogous to an ear-drum per-. forated by blast. But why the retinal detachment figures are so ridiculously low with so many other manifestations of blast in the body I am unable to determine.

Sympathetic ophthalmia has not been seen by. me in military practice, despite the fact that I have had no facilities for slit-lamp microscopy.

The visual disorders; Table III, show a very high proportion of refractive errors, and disclose a number of visually $B$ class men who have slipped through the rushed recruiting in Australia. It still further stresses the necessity of recruits with less than $6 / 9$ vision in either eye being submitted to an ophthalmic examination before acceptance for overseas service.

The muscle anomalies (Table IV) were heavy, and many needed treatment. The result of these treatments will be recorded in a special report by me on this subject entitled " orthoptics in the field." Of more than passing significance were 17 cases of overaction of the inferior oblique, which cases I feel must be analysed at a later date. 
The virus keratitis cases were very. troublesome, and on the whole had a higher incidence than in Australia. I have written notes on these specific conditions in the Middle East, and they are to be published at a later date under the title " notes on forms of keratitis presumably due to the virus of herpes simplex." Suffice here to say that any pyrexia (malaria, relapsing fever, dysentery, or even T.A.B. injections) precipitate an attack of virus keratitis in one or both eyes, and that in the hot climate of the Middle East a virus corneal infection is difficult to eradicate (see Table V).

Glare asthenopia at 4.153 per cent. of cases and illumination deficiencies of 0.700 per cent. call for special comment, for none is recorded in my Tasmanian figures. The extremes of illumination manifest in the Middle East are responsible for the high eye consultation rate in the A.I.F. From desert glare when driving by day to black-out driving by night or to hurricane lamp for reading produces a contrast intolerable to many eyes. I have strongly advocated goggles for driving and petrol lamps for reading, and I must thank the Australian Red Cross for their continued help in the matter of goggles for the troops.

Pterygia showed a high incidence in the A.I.F., but then this disease is very prevalent both in tropical Australia and tropical Levant, while in Tasmania it is of little account. Nevertheless, in the Middle East very few pterygia required operative treatment, and when they were marsupialised the operation field remained irritable for months.

The amblyopias need to be mentioned. There are as many amblyopias ex anopsia, in army personnel as in civil life, 3.696 per cent. to 3.778 per cent. respectively, which again stresses too rapid recruiting. The functional amblyopias, on the other hand, are four times as common, which is only to be expected. The discrepancy between army and civil figures in congenital amblyopia I cannot explain.

I pointed out in my article of January 10,1942 , that hypopyon ulcer or hypopyon iritis was very rare in Tasmania, namely, 0.1 per cent. But in the army it is almost unknown at 0.084 per cent. This low military figure-is possibly due to the early exhibition of sulphanilamide or its derivatives by mouth-for none of my patients had local application of these substances, as they were not procurable.

Iridocyclitis is extremely rare in the army, and four out of five of the cases were due to spirochaetal relapsing fever, detailed in " The ocular complication of relapsing fever," in course of publiçation. These four cases all recovered normal vision spontaneously and were not influenced by arsenic.

Anisocoria of non-Argyll Robertson and non-myotonic type is twice as.prevalent in the army as in civil life. This is not due 
to syphilis, as I have proved by many Kline tests, but is due to a high incidence of sandfly fever and anxiety-state. Although these two diseases are aetiologically poles apart, they nevertheless seem to precipitate a similar type of anisocoria-that is, one which is transient and not permanent, and does not affect pupillary reactions to light and convergence.

I ropical or Egyptian ophthalmia was not once found in the whole series. This is astounding when it is realised that this was the major factor in bringing ruin to Napoleon's Egyptian campaign in 1800 A.D., and was the cause of some anxiety in the 1914-1918 campaign in the Middle East. . Trachoma likewise was not contracted in one instance in the Middle East in my 3,638 patients, the whole of the 16 army cases having had their original infection in Australia prior to enlistment. Certainly hygiene was - far better in 1941-1942 than in 1800, but with many military camps in 1941-1942 in close contact to Arab villages, in a country where trachoma and ophthalmia are rife, it makes one wonder how immunity to these dangers has changed so rapidly in 140 years.

Finally, those patients without any disease to account for symptoms. These figures (like functional amblyopia) were three times as high in the A.I.F. as they. were in civil practice, but to find only 200 unnecessary consultations out of 3,638 soldiers reflects the greatest credit on the sagacity of the Unit R.M.Os. It is appropriate to remark here on the close co-operation prevailing in all branches of the A.A.M.C. and on the excellent consultation service always available to the men of the A.I.F.

In conclusion, I should like to thank the ophthalmic staff' of an Australian General Hospital for their help with these figures, which have had my personal supervision throughout. Colonel J. G. Hayden, who commands this hospital, has given his permission for these figures to be published, for which I thank him also.

\section{References}

- Except for my article on "Eye Disease in Tasmania," published in the Medical Journal of Australia on January 10, 1942, there is no available literature at my disposal on this subject. My articles on "The ocular complication of relapsing fever," " notes on forms of keratitis presumable due to the virus of herpes simplex," and " orthoptics in the field " are in the process of publication either in the British Journal of Ophthalmology or in the Medical Journal of Australia. 
TABLE I

COMPARATIVE TABLES OF EYE DISEASES, ETC.

1. Private Practice, Hobart 1931-1939. 6458 patients, showing 14,317 diseased conditions, 239 diseases.

2. 7th A.G.H., Middle East, May 1941-November 1942. 3638 Australian patients, 5650 diseased conditions, 226 diseases.

\begin{tabular}{|c|c|c|c|c|c|c|c|}
\hline List of Diseas & es, etc & & $\therefore$ & Civil & Per cent. & War & Per cent. \\
\hline Abscess of brow & $\therefore$ & $\ldots$ & $\ldots$ & 18 & $0 \cdot 279^{\circ}$ & - & - \\
\hline Abscess of caruncle & & $\cdots$ & $\ldots$ & - & - & 1 & 0.027 \\
\hline Accommodation, paraly & sis of & $\ldots$ & $\ldots$ & 37 & $0 \cdot 573$ & 10 & 0.275 \\
\hline Albinism $\ldots . .$. & $\ldots$ & $\ldots$ & $\ldots$ & 5 & $0 \cdot 077$ & - & - \\
\hline Alopecia of eyebrow & ... & $\ldots$ & $\ldots$ & - & - & 1 & $0 \cdot 027$ \\
\hline Amblyopia, congential & ... & $\ldots$ & $\ldots$ & 35 & $0 \cdot 542$ & 5 & $0 \cdot 137$ \\
\hline Amblyopia ex anopsia & $\ldots$ & $\ldots$ & $\ldots$ & 244 & $3 \cdot 778$ & 132 & $3 \cdot 628$ \\
\hline Amblyopia, functional & & $\ldots$ & $\ldots$ & 14 & $0 \cdot 217$ & 32 & $0 \cdot 879$ \\
\hline Anaesthesia of cornea & $\ldots$ & $\therefore$ & $\cdots$ & 3 & $0 \cdot 046$ & 1 & 0.027 \\
\hline Anaesthesia of orbital $n$ & terves & $\ldots$ & $\ldots$ & 3 & $0 \cdot 046$ & 3 & 0.082 \\
\hline Angioid streaks $\ldots$ & $\ldots$ & $\ldots$ & $\ldots$ & 4 & 0.062 & - & - \\
\hline Angioma $\ldots \quad \ldots$ & $\ldots$ & $\ldots$ & $\ldots$ & $\mathbf{5}$ & 0.077 & 6 & $0 \cdot 165$ \\
\hline Angioneurotic oedema & $\ldots$ & $\ldots$ & $\ldots$ & 2 & 0.031 & 1 & 0.027 \\
\hline Anisocoria $\quad \ldots$ & $\ldots$ & $\ldots$ & $\cdots$. & 91 & $1 \cdot 409$ & 92 & $2 \cdot 529$ \\
\hline Anisometropia & $\ldots$ & $\ldots$ & $\ldots$ & 82 & $1 \cdot 270$ & 38 & $1 \cdot 045$ \\
\hline Anophthalmos $\quad \ldots$ & $\ldots$ & $\ldots$ & $\ldots$ & 83 & $1 \cdot 285$ & 24 & $0: 660$ \\
\hline Anopia, quadrantic and & hemi. & & $\ldots$ & 16 & $0 \cdot 248$ & 4 & $0 \cdot 110$ \\
\hline Aphakia $\ldots . \quad \ldots$ & $\ldots$ & ... & $\ldots$ & 153 & $2 \cdot 369$ & 6 & $0 \cdot 165$ \\
\hline Argyll Robertson pupil & & $\ldots$ & $\ldots$ & 5 & 0.077 & 2 & $0 \cdot 054$ \\
\hline Argyrosis ... $\ldots$ & $\ldots$ & $\ldots$ & $\ldots$ & 10 & 0.155 & - & 一. \\
\hline Atropine irritation & $\ldots$ & $\ldots$ & $\ldots$ & 12 & $0: 186$ & 4 & $0 \cdot 110$ \\
\hline Birth Injuries $\ldots$ & $\ldots$ & $\ldots$ & $\ldots$ & 34. & 0.526 & 1 & $0 \cdot 027$ \\
\hline Blepharitis, simple & $\ldots$ & $\ldots$ & $\ldots$ & 153 & $2 \cdot 369$ & 112 & $3 \cdot 079$ \\
\hline Blepharitis, ulcerative & $\ldots$ & $\ldots$ & $\ldots$ & 7 & $0 \cdot 108$ & 6 & $0 \cdot 165$ \\
\hline Blepharospasm, functio & nal & $\ldots$ & $\ldots$ & - & - & 1 & $0 \cdot 027$ \\
\hline Burn of conjunctiva & $\ldots$ & $\ldots$ & $\ldots$ & 10 & $0 \cdot 155$ & 8 & $0 \cdot 220$ \\
\hline Burn of cornea $\quad .$. & $\ldots$ & $\ldots$ & $\ldots$ & 12 & $0 \cdot 186$ & 4 & $0 \cdot 110$ \\
\hline Burn of lid $\quad \ldots$ & $\ldots$ & $\ldots$ & $\ldots$ & - & - & 3 & $0 \cdot 082$ \\
\hline Burn of retina $\quad \ldots$ & $\ldots$ & $\ldots$ & $\ldots$ & - & - & 2 & $0 \cdot 055$ \\
\hline Canaliculus, laceration & & $\ldots$ & $\ldots$ & 3 & 0.046 & $\overline{3}$ & 0.082 \\
\hline Cataract, anterior polar & & $\ldots$ & $\therefore$. & 4 & 0.062 & 3 & 0.082 \\
\hline Cataract, blue dot & $\ldots$ & $\ldots$ & $\ldots$ & 11 & $0 \cdot 170$ & 2 & 0.055 \\
\hline Cataract, complicated & $\ldots$. & $\ldots$ & $\ldots$ & 50 & $0 \cdot 774$ & 5 & $0 \cdot 137$ \\
\hline Cataract, congenital & $\ldots$ & $\ldots$ & $\ldots$ & 64 & 0.991 & 8 & $0 \cdot 220$ \\
\hline ct, diabetic & $\ldots$ & $\ldots$ & $\ldots$ & 38 & $0 \cdot 588$ & - & - \\
\hline Cataract, glassblowers' & $\ldots$ & $\cdots$ & $\ldots$ & 1 & 0.015 & - & - \\
\hline Cataract, lamellar & $\ldots$ & $\ldots$ & $\ldots$ & 5 & 0.077 & 2 & $0 \cdot 055$ \\
\hline Cataract, senile $\ldots$ & $\ldots$ & & $\ldots$ & 669 & $10 \cdot 359$ & 6 & $0 \cdot 165$ \\
\hline Cataract, traumatic & $\ldots$ & & $\ldots$ & 54 & 0.836 & 28 & $0 \cdot 770$ \\
\hline Cataract, X-ray ... & $\ldots$ & $\ldots$ & $\ldots$ & 1 & 0.015 & - & - \\
\hline Chalazion ... $\quad \ldots$ & $\therefore$ & $\ldots$ & $\ldots$ & 112 & $1 \cdot 734$ & 68 & $1 \cdot 869$ \\
\hline Choroid, atrophy (post & traum & & $\ldots$ & - & - & 2 & $0 \cdot 055$ \\
\hline Choroid, detachment of & & $\ldots$ & $\ldots$ & 1 & 0.015 & - & - \\
\hline Choroid, rupture of & $\ldots$ & $\ldots$ & $\cdots$ & 5 & 0.077 & $\mathbf{3}$ & $0 \cdot 082$ \\
\hline Choroid, sarcoma of & $\ldots$ & & & 11 & $0 \cdot 170$ & 1 & $0 \cdot 027$ \\
\hline Choroid, stretching of & $\ldots$ & $\ldots$ & $\ldots$ & 39 & $0 \cdot 604$ & - & - \\
\hline Choroiditis, acute & $\ldots$ & $\ldots$ & $\therefore$ & 9 & $0 \cdot 139$ & 2 & 0.055 \\
\hline Choroiditis, Tay's & $\ldots$ & $\ldots$ & & 63 & 0.975 & 14 & $0 \cdot 385$ \\
\hline Choroido-retinitis & $\ldots$ & $\ldots$ & $\ldots$ & 168 & $2 \cdot 601$ & 90 & $2 \cdot 474$ \\
\hline Coloboma of retina & $\ldots$ & $\ldots$ & $\ldots$ & 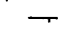 & - & 1 & 0.027 \\
\hline Colobomata of choroid & & & $\ldots$ & 4 & 0.062 & $\rightarrow$ & $\therefore$ \\
\hline
\end{tabular}


TABLE I-continued

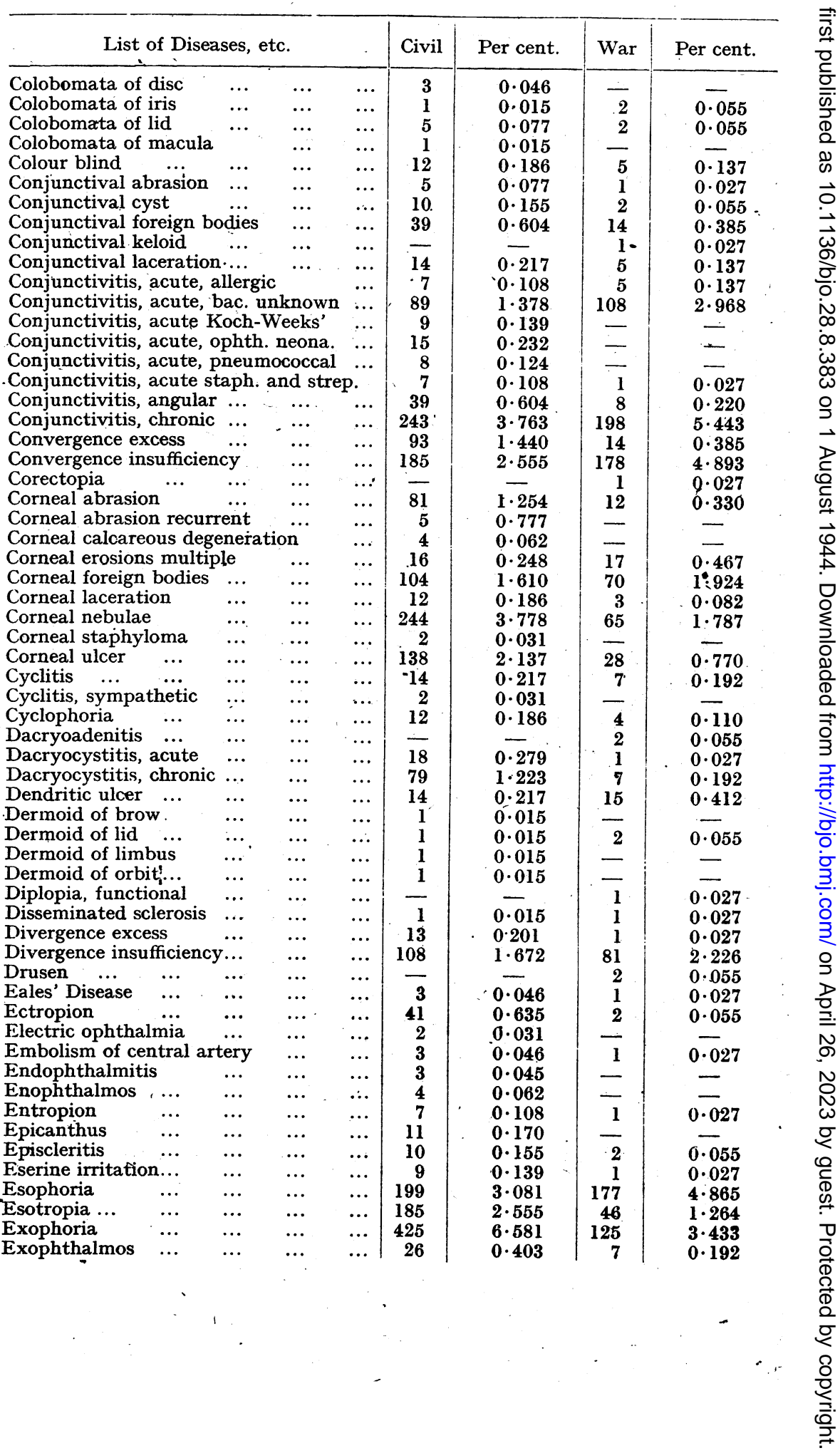


TABLE I-continued

\begin{tabular}{|c|c|c|c|c|c|c|c|}
\hline \multicolumn{4}{|c|}{ List of Diseases, etc. } & Civil & Per cent. & War & Per cent. \\
\hline Exotropia & & .. & $\cdots$ & 89 & $1 \cdot 378$ & 29 & $0 \cdot 797$ \\
\hline Furuncle of caruncle & $\ldots$ & $\ldots$ & $\ldots$ & 一 & - & 2 & 0.055 \\
\hline Glare asthenopia & $\cdots$ & $\ldots$ & $\cdots$ & - & - & 151 & $4 \cdot 151$ \\
\hline Glaucoma, acute ... & $\ldots$ & $\ldots$ & $\ldots$ & 8 & $0 \cdot 124$ & 1 & 0.027 \\
\hline Glaucoma, chronic & $\ldots$ & ... & $\ldots$ & 78 & $1 \cdot 208$ & 2 & 0.055 \\
\hline Glaucoma, secondary & $\ldots$ & $\ldots$ & $\ldots$ & 50 & $0 \cdot 774$ & 3 & 0.082 \\
\hline Glioma retinae $\quad \ldots$ & $\ldots$ & $\ldots$ & $\ldots$ & 2 & 0.031 & - & - \\
\hline Globe, contusion of & $\ldots$ & $\ldots$ & $\ldots$ & 38 & 0.588 & 5 & $0 \cdot 137$ \\
\hline Globe, perforation of & ... & $\ldots$ & $\ldots$ & 64 & 0.991 & 11 & $0 \cdot 302$ \\
\hline Haemangioma $\quad \ldots$. & $\ldots$ & $\ldots$ & $\ldots$ & 9 & $0 \cdot 124$ & 1 & $0 \cdot 027$ \\
\hline Haemorrhage, retinal & $\ldots$ & $\ldots$ & $\ldots$ & 39 & $0 \cdot 604$ & $\rightarrow$ & - \\
\hline Haemorrhage, subconju & unctival & & $\ldots$ & 62 & $0 \cdot 960$ & 20 & 0.550 \\
\hline Haemorrhage, subhyalc & oid & $\ldots$ & $\ldots$ & 3 & $0 \cdot 046$ & 1 & $a \cdot 027$ \\
\hline Herpes ophthalmious & $\ldots$ & $\cdots$ & $\ldots$ & 18 & $0 \cdot 279$ & - & - \\
\hline Heterochromia $\ldots$ & $\ldots$ & $\ldots$ & $\ldots$ & 9 & $0 \cdot 139$ & 2 & 0.055 \\
\hline Hordeolum , ... & $\ldots$ & $\ldots$ & $\ldots$ & 180 & $2 \cdot 478$ & 123 & $\mathbf{3} \cdot \mathbf{3 5 3}$ \\
\hline Horner's syndrome & ... & $\cdots$ & $\ldots$ & 4 & $0 \cdot 062$ & 2 & 0.055 \\
\hline Hyaloid, persistent & $\ldots$ & $\ldots$ & $\ldots$ & 7 & $0 \cdot 108$ & 5 & $0 \cdot 137$ \\
\hline Hypermetropia $\ldots$ & $\because$ & $\ldots$ & $\ldots$ & 260 & $5 \cdot 543$ & 234 & $6 \cdot 432$ \\
\hline Hypermetropic astigma & atism & ... & $\cdots$ & 1949 & $30 \cdot 149$ & 1044 & $28 \cdot 697$ \\
\hline Hyperphoriă $\quad \ldots$ & ... & $\cdots$ & ... & 107 & $1 \cdot 657$ & 23 & $0 \cdot 632$ \\
\hline Hyphaema & $\cdots$ & $\cdots$ & $\cdots$ & 28 & $0 \cdot 434$ & 15 & 0.412 \\
\hline Hypopyon , $\quad .$. & $\cdots$ & $\cdots$ & $\cdots$ & 11 & $0 \cdot 170$ & 3 & $0 \cdot 082$ \\
\hline Hypothyroidism ... & $\cdots$ & $\cdots$ & $\cdots$ & 3 & $0 \cdot 046$ & - & - \\
\hline Icteroid conjunctiva & $\ldots$ & $\cdots$ & $\cdots$ & - & - & 3 & 0.082 \\
\hline Illumination deficiency & & ... & ... & $\overline{10}$ & $=$ & 25 & 0.687 \\
\hline Intracranial tumour & & $\ldots$ & $\ldots$ & 17 & $0 \cdot 263$ & 1 & $0 \cdot 027$ \\
\hline Intraocular foreign bod & dies & ... & ... & 10 & $0 \cdot 155$ & 17 & $0 \cdot 467$ \\
\hline Iris, atrophy of $\ldots$ & $\ldots$ & $\cdots$ & $\ldots$ & 5 & $0 \cdot 077$ & - & 一 \\
\hline Iris, bombé . ... & $\cdots$ & $\cdots$ & $\cdots$ & 11 & $0 \cdot 170$ & - & - \\
\hline Iris, prolapse of $\ldots$ & $\cdots$ & $\cdots$. & $\cdots$ & 12 & $0 \cdot 186$ & 5 & $0 \cdot 137$ \\
\hline Iris, perforation of & $\cdots$ & $\cdots$ & $\cdots$ & $\mathbf{5}$ & $0 \cdot 077$ & - & - \\
\hline Iris, sphincter rupture & $\ldots$ & ... & $\ldots$ & 6 & $0 \cdot 093$ & 3 & 0.082 \\
\hline Iridocyclitis $\quad \ldots$ & $\cdots$ & $\cdots$ & $\therefore$ & 55 & 0.851 & $\mathbf{5}$ & $0 \cdot 137$ \\
\hline Iridodialysis $\quad \ldots$ & $\cdots$ & $\cdots$ & $\ldots$ & 9 & $0 \cdot 139$ & 1 & $0: 027$ \\
\hline Iritis, acute & $\cdots$ & $\cdots$ & $\cdots$ & 44 & $0 \cdot 681$ & 7 & $0 \cdot 192$ \\
\hline Iritis, diabetic $\quad \ldots$ & $\cdots$ & $\cdots$ & $\cdots$ & 5 & $0 \cdot 077$ & - & - \\
\hline Iritis, old $\quad \ldots$ & $\cdots$ & $\cdots$ & $\cdots$ & 26 & $0 \cdot 403$ & $\mathbf{5}$ & $0 \cdot 137$ \\
\hline Jaw blinking $\quad \ldots$ & $\cdots$ & $\cdots$ & $\cdots$ & - & - & 1 & $0 \cdot 027$ \\
\hline Keratitis (unspecified) & $\ldots$ & $\cdots$ & $\cdots$ & 16 & $0 \cdot 243$ & 9 & $0 \cdot 247$ \\
\hline Keratitis acne rosacea & & $\therefore$ & $\cdots$ & 11 & $0 \cdot 170$ & - & - \\
\hline Keratitis bullous & $\cdots$ & $\cdots$ & $\cdots$ & 4 & 0.062 & - & - \\
\hline Keratitis disciform & $\cdots$ & $\cdots$ & $\cdots$ & 3 & $0 \cdot 046$ & 5 & $0 \cdot 137$ \\
\hline Keratitis filamentary & $\cdots$ & $\cdots$ & $\cdots$ & 3 & $0 \cdot 046$ & $\overline{9}$ & $\overline{0} 00$ \\
\hline & $\therefore$ & $\cdots$ & $\cdots$ & $\overrightarrow{29}$ & $\overline{0.449}$ & 3 & $\begin{array}{l}0.082 \\
0.165\end{array}$ \\
\hline & $\cdots$ & $\cdots$ & & $\begin{array}{r}29 \\
5\end{array}$ & $\begin{array}{l}0 \cdot 449 \\
0 \cdot 077\end{array}$ & 2 & $\begin{array}{l}0.165 \\
0.055\end{array}$ \\
\hline $\begin{array}{l}\text { Keratitis lagophthalmo } \\
\text { Keratitis marginal }\end{array}$ & & $\cdots$ & & $\begin{array}{r}5 \\
60\end{array}$ & 0.929 & 47 & $\begin{array}{l}0.000 \\
1 \cdot 292\end{array}$ \\
\hline & $\cdots$ & $\begin{array}{l}\cdots \\
\cdots\end{array}$ & & $\begin{array}{r}00 \\
2\end{array}$ & 0.031 & - & 1.202 \\
\hline $\begin{array}{l}\text { Keratitis mustard gas } \\
\text { Keratitis neuroparalyti }\end{array}$ & & $\cdots$ & & 9 & $0 \cdot 139$ & - & - \\
\hline Keratitis profun̨da & $\ldots$ & & & 12 & 0.186 & 1 & 0.027 \\
\hline Keratitis, striate & ... & ... & & 8 & 0.124 & $\mathbf{l}$ & 0.027 \\
\hline Keratitis, superficial & $\ldots$ & $\ldots$ & ... & 15 & 0.232 & $\overline{2}$ & 0.055 \\
\hline Keratitis, superficial pu & anctate & & $\ldots$ & 95 & $1 \cdot 471$ & 37 & $1 \cdot 017$ \\
\hline Keratitis, vesicular & $\ldots$ & ... & $\ldots$ & 11 & $0 \cdot 170$ & $\theta$ & $0 \cdot 247$ \\
\hline Keratoconus ... & $\ldots$ & ... & ... & 32 & $0 \cdot 495$ & 2 & 0.055 \\
\hline Keratoglobus $\quad \ldots$ & $\ldots$ & $\ldots$ & $\ldots$ & - & - & 1 & 0.027 \\
\hline Lacrimal fistula ... & $\ldots$ & ... & ... & $\mathbf{3}$ & $0 \cdot 046$ & 1 & $0 \cdot 027$ \\
\hline Lashes, itinerant & $\ldots$ & .. & ... & 8 & $0 \cdot 124$ & - & - \\
\hline Lens, dislocation of & $\ldots$ & $\ldots$ & $\ldots$ & 15 & 0.232 & 3 & 0.082 \\
\hline
\end{tabular}


TABLE I-continued




TABLE I-continued

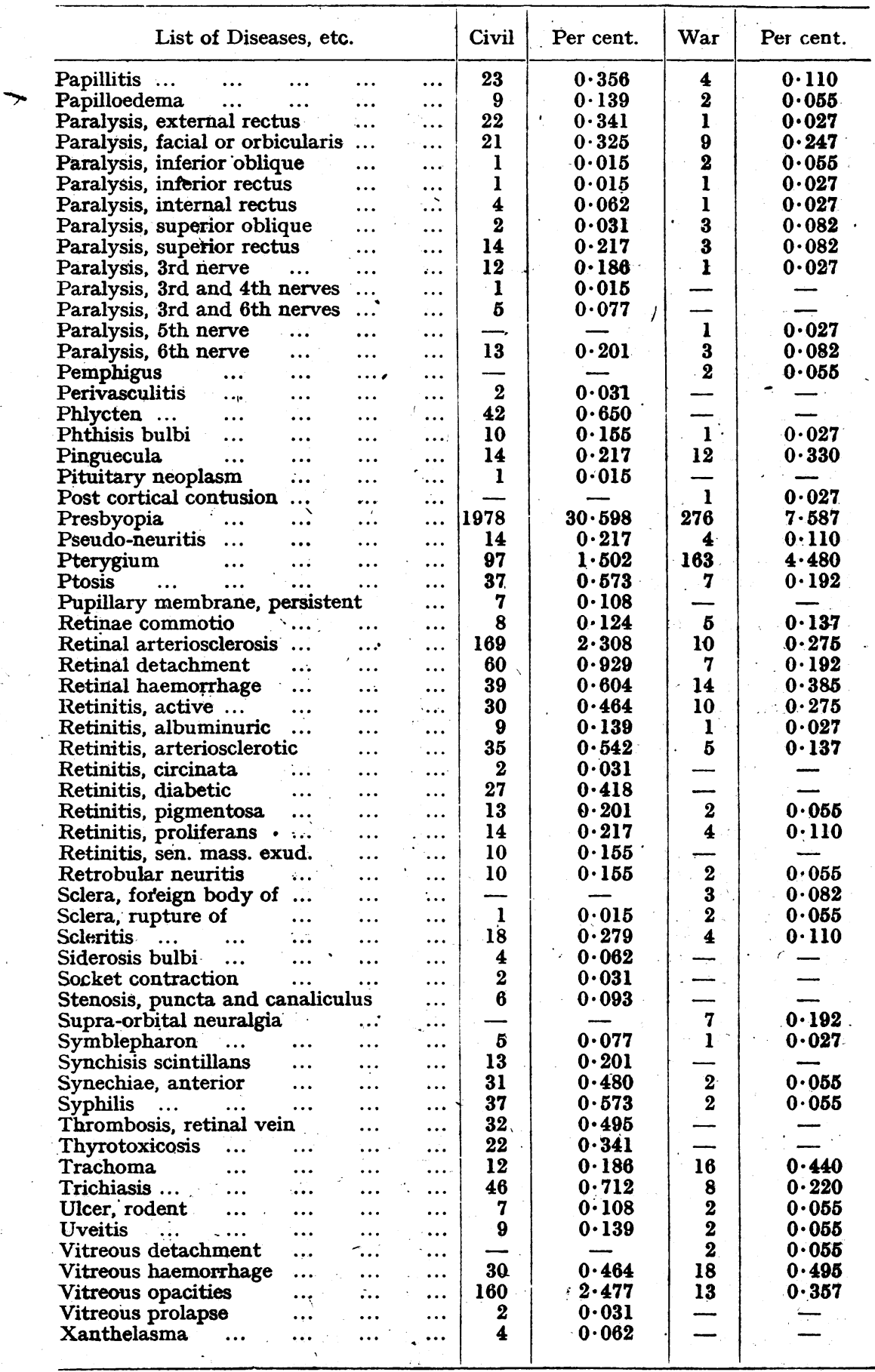


TABLE II

COMPARATIVE LIST OF .EYE INJURIES (see Table I)

\begin{tabular}{|c|c|c|c|c|c|c|c|}
\hline \multicolumn{3}{|c|}{ Injuries } & & Civil & Per cent. & War & Per cent. \\
\hline 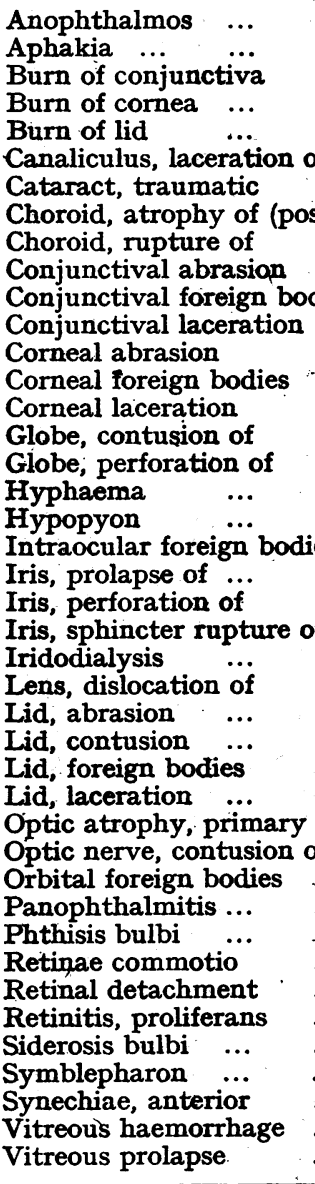 & 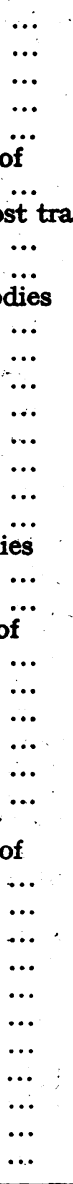 & 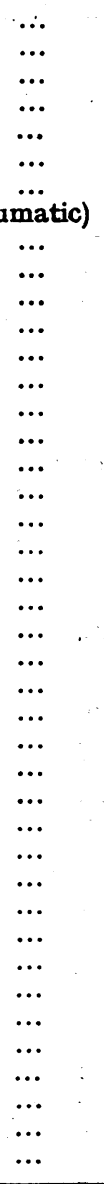 & 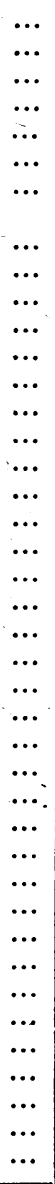 & $\begin{array}{r}83 \\
153 \\
10 \\
12 \\
-\frac{1}{3} \\
54 \\
5 \\
5 \\
39 \\
14 \\
81 \\
104 \\
12 \\
38 \\
64 \\
28 \\
11 \\
10 \\
12 \\
5 \\
6 \\
9 \\
15 \\
4 \\
16 \\
6 \\
11 \\
30 \\
2 \\
3 \\
10 \\
10 \\
60 \\
14 \\
4 \\
5 \\
31 \\
30 \\
2\end{array}$ & $\begin{array}{c}1 \cdot 285 \\
-2 \cdot 369 \\
0.155 \\
0.186 \\
- \\
0.046 \\
0.836 \\
- \\
0.077 \\
0.077 \\
0.604 \\
0.217 \\
1.254 \\
1.610 \\
0.186 \\
0.588 \\
0.991 \\
0.434 \\
0.170 \\
0.155 \\
0.186 \\
0.077 \\
0.093 \\
0.139 \\
0.232 \\
0.062 \\
0.248 \\
0.093 \\
0.170 \\
0.464 \\
- \\
0.031 \\
0.046 \\
0.155 \\
0.124 \\
0.929 \\
0.217 \\
0.062 \\
0.077 \\
0.480 \\
0.464 \\
0.031\end{array}$ & $\begin{array}{r}24 \\
6 \\
8 \\
4 \\
3 \\
3 \\
28 \\
2 \\
2 \\
3 \\
1 \\
14 \\
5 \\
12 \\
70 \\
3 \\
5 \\
11 \\
15 \\
3 \\
17 \\
5 \\
3 \\
1 \\
3 \\
8 \\
5 \\
26 \\
3 \\
2 \\
10 \\
1 \\
1 \\
5 \\
7 \\
4 \\
1 \\
2 \\
18 \\
- \\
\end{array}$ & $\begin{array}{l}0 \cdot 660 \\
0 \cdot 165 \\
0 \cdot 220 \\
0 \cdot 110 \\
0 \cdot 082 \\
0 \cdot 082 \\
0 \cdot 770 \\
0 \cdot 055 \\
0 \cdot 082 \\
0 \cdot 027 \\
0 \cdot 385 \\
0 \cdot 137 \\
0 \cdot 330 \\
1 \cdot 924 \\
0 \cdot 082 \\
0 \cdot 137 \\
0 \cdot 302 \\
0 \cdot 412 \\
0 \cdot 082 \\
0 \cdot 467 \\
0 \cdot 137 \\
0 \cdot 082 \\
0 \cdot 027 \\
0 \cdot 082 \\
0 \cdot- \\
0 \cdot 220 \\
0 \cdot 137 \\
0 \cdot 715 \\
0 \cdot 082 \\
0 \cdot 055 \\
0 \cdot 275 \\
0 \cdot 027 \\
0 \cdot 027 \\
0 \cdot 137 \\
0 \cdot 192 \\
0 \cdot 110 \\
0 \cdot 027 \\
0 \cdot 055 \\
0 \cdot 495 \\
-.\end{array}$ \\
\hline $\begin{array}{l}39 \text { headings (Civil) } \\
38 \text { headings (War) }\end{array}$ & $\begin{array}{l}\cdots \\
\cdots\end{array}$ & $\cdots$ & $\begin{array}{l}\cdots \\
\cdots\end{array}$ & 1009 & $15 \cdot 624$ & 342 & $9 \cdot 400$ \\
\hline
\end{tabular}

TABLE III

COMPARATIVE LIST OF VISUAL DISORDERS (see Table I)

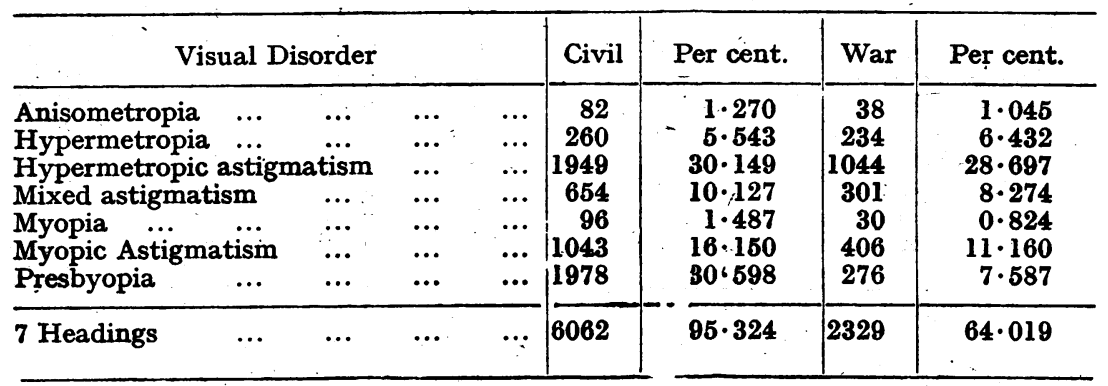




\section{TABLE IV}

\section{COMPARATIVE LIST OF MUSCULAR ANOMALIES}

(see Table I)

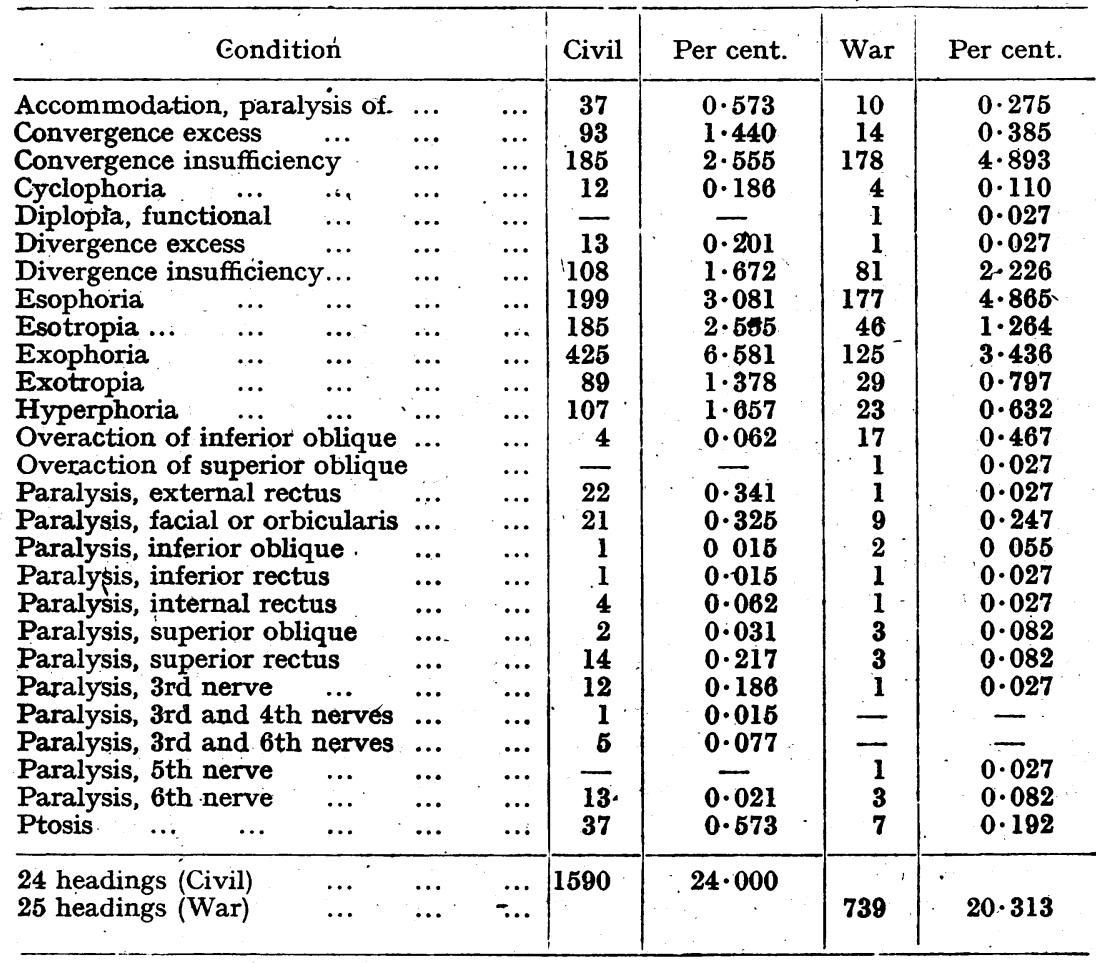

TABLE V

COMPARATIVE LIST OF KERATITIS (see Table I)

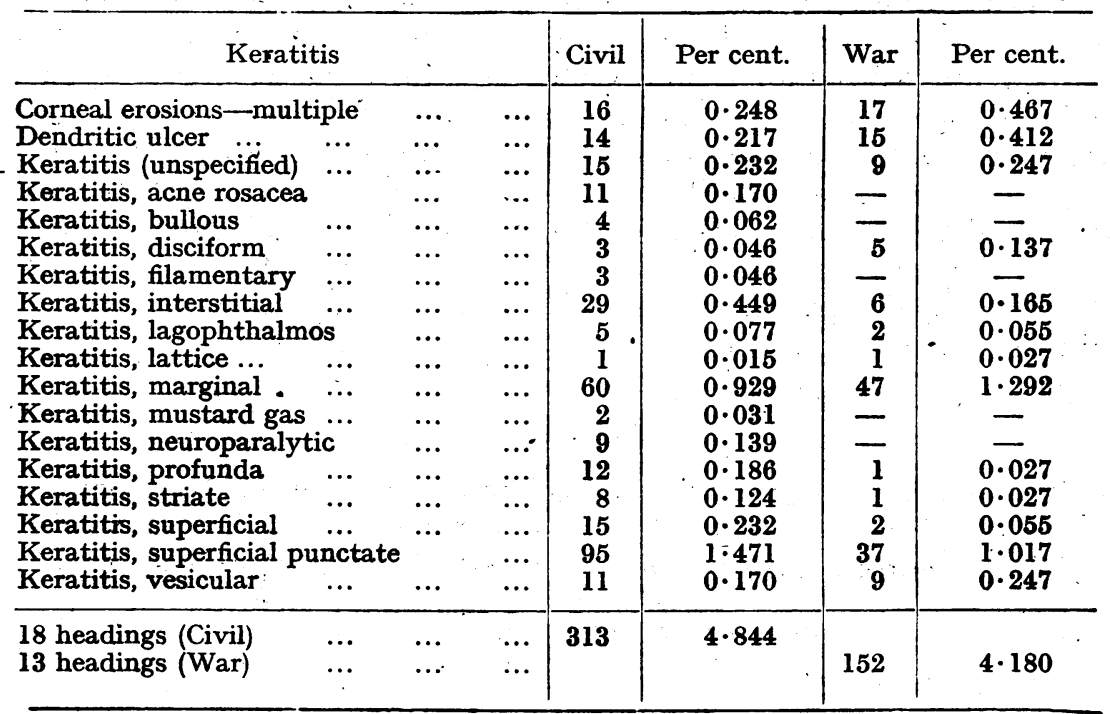

